\title{
HEAT KERNELS IN THE CONTEXT OF KATO POTENTIALS ON ARBITRARY MANIFOLDS
}

\author{
BATU GÜNEYSU
}

\begin{abstract}
By introducing the concept of Kato control pairs for a given Riemannian minimal heat kernel, we prove that on every Riemannian manifold $(M, g)$ the Kato class $\mathcal{K}(M, g)$ has a subspace of the form $\mathrm{L}^{q}(M, \mathrm{~d} \varrho)$, where $\varrho$ has a continuous density with respect to the volume measure $\mu_{g}$ (where $q$ depends on $\operatorname{dim}(M)$ ). Using a local parabolic $\mathrm{L}^{1}$-mean value inequality, we prove the existence of such densities for every Riemannian manifold, which in particular implies $\mathrm{L}_{\text {loc }}^{q}(M) \subset \mathcal{K}_{\text {loc }}(M, g)$. Based on previously established results, the latter local fact can be applied to the question of essential selfadjointness of Schrödinger operators with singular magnetic and electric potentials. Finally, we also provide a Kato criterion in terms of minimal Riemannian submersions.
\end{abstract}

\section{INTRODUCTION}

Given a Riemannian manifold $(M, g)$ with $\mu_{g}$ the Riemannian volume measure, a Borel function $w: M \rightarrow \mathbb{R}$ is said to be Kato class of $(M, g)$, symbolically $w \in \mathcal{K}(M, g)$, if

$$
\lim _{t \rightarrow 0+} \sup _{x \in M} \int_{0}^{t} \int_{M} \mathrm{e}^{(s / 2) \Delta_{g}}(x, y)|w(y)| \mathrm{d} \mu_{g}(y) \mathrm{d} s=0,
$$

where $(-1 / 2) \Delta_{g} \geq 0$ denotes the Friedrichs realization of $(1 / 2)$ times the Laplace-Beltrami operator in $\mathrm{L}^{2}\left(M, \mathrm{~d} \mu_{g}\right)$. In particular, $\mathrm{e}^{\frac{s}{2} \Delta_{g}}(x, y)$ is precisely the minimal nonnegative heat kernel $p_{g}(s, x, y)$ on $(M, g)$. Likewise, there is the local counterpart $\mathcal{K}_{\text {loc }}(M, g)$, which is given by all $w$ such that $1_{K} w \in \mathcal{K}(M, g)$ for all compact $K \subset M$. Ever since its introduction, the Kato class has proved to be a convenient and large class of perturbations of $(-1 / 2) \Delta_{g}$, for which the following important results hold true simultaniously: For every $w=w_{+}-w_{-}$such that its positive part satisfies $w_{+} \in \mathrm{L}_{\text {loc }}^{1}(M)$, its negative part satisfies $w_{-} \in \mathcal{K}(M, g)$,

I) $w_{-}$is an infinitesimally small perturbation of $(-1 / 2) \Delta_{g}$ (cf. [19]) in the sense of quadratic forms; in particular the form sum $H_{g}^{w}=(-1 / 2) \Delta_{g} \pm w$ is a well-defined self-adjoint operator in $\mathrm{L}^{2}\left(M, \mathrm{~d} \mu_{g}\right)$ which is bounded from below

II) one has $\mathrm{L}^{q}\left(M, \mathrm{~d} \mu_{g}\right) \rightarrow \mathrm{L}^{q}\left(M, \mathrm{~d} \mu_{g}\right)$-bounds of the form (cf. Proposition A.1 below)

$$
\left\|\mathrm{e}^{-t H_{g}^{w}}\right\|_{\mathrm{L}^{q}\left(M, \mathrm{~d} \mu_{g}\right) \rightarrow \mathrm{L}^{q}\left(M, \mathrm{~d} \mu_{g}\right)} \leq \delta \mathrm{e}^{t C\left(\delta, w_{-}, g\right)} \text {, for every } \delta>1
$$

III) $x \mapsto \mathrm{e}^{-t H_{g}^{w}} f(x)$ is continuous [18] for all $f \in \mathrm{L}^{\infty}\left(M, \mathrm{~d} \mu_{g}\right)[18]$

The remarkable fact about these results is that all of them do not require any additional assumptions on the Riemannian structure $g$ on $M$. The bound from II) with $q=\infty$ has been used recently in the context of the Riemannian total variation by D. Pallara and 
the author in [8]. Let us also note that one can even establish a semigroup theory of perturbations given by Kato measures rather than Kato functions: Here there exist very subtle results by Sturm [20], Stollmann-Voigt [19], and Kuwae-Takahashi [14], and can even do more general than that [18].

There is another important result which is built on the local Kato class [7]:

IV) If $(M, g)$ is geodesically complete, if $\alpha \in \Gamma_{\mathrm{L}_{\text {loc }}^{4}}\left(M, T^{*} M\right)$ is a magnetic potential with $\operatorname{grad}(\alpha) \in \mathrm{L}_{\text {loc }}^{2}(M)$, and if $w \in \mathcal{K}_{\text {loc }}(M, g) \cap \mathrm{L}_{\text {loc }}^{2}(M)$ is an electric potential such that the corresponding magnetic Schrödinger operator $H_{g}^{\alpha, w}$ is bounded from below on the smooth compactly supported functions, then $H_{g}^{\alpha, w}$ is in fact essentially self-adjoint.

Apart from the above "success" of the Kato class from an abstract point of view, as one knows the explicit form of $p_{g}(t, x, y)$ only in very few cases, the following question remains: When is a given Borel function $w$ on $M$ actually in $\mathcal{K}(M, g)$ or in $\mathcal{K}_{\text {loc }}(M, g)$ ?

In the Euclidean $\mathbb{R}^{m}$ this question is usually easy to answer, as one has the characterization $w \in \mathcal{K}\left(\mathbb{R}^{m}\right)$, if and only if

$$
\begin{aligned}
& w \in \mathrm{L}_{\text {unif,loc }}^{1}(\mathbb{R}), \quad \text { if } m=1, \\
& \lim _{r \rightarrow 0+} \sup _{x \in \mathbb{R}^{m}} \int_{|x-y| \leq r}|w(y)| h_{m}(|x-y|) \mathrm{d} y, \quad \text { if } m \geq 2,
\end{aligned}
$$

where $h_{m}:[0, \infty] \rightarrow[0, \infty]$ is given by

$$
h_{2}(r):=\log ^{+}(1 / r), h_{m}(r):=r^{2-m}, \text { if } m>2,
$$

In fact Kato has introduced $\mathcal{K}\left(\mathbb{R}^{m}\right)$ essentially in this "analytic" form in [13], and the equivalence of the latter definition to the above heat-kernel definition has been shown by Aizenman-Simon in [1]. The characterization $(1)+(2)$ straightforwardly implies the inclusions $^{1}$

$$
\mathrm{L}^{q}\left(\mathbb{R}^{m}\right) \subset \mathcal{K}\left(\mathbb{R}^{m}\right) \text { if } q \geq 1 \text { in case } m=1 \text {, and if } q>m / 2 \text { in case } m \geq 2,
$$

which of course implies $\mathrm{L}_{\text {loc }}^{q}\left(\mathbb{R}^{m}\right) \subset \mathcal{K}_{\text {loc }}\left(\mathbb{R}^{m}\right)$. The proof of the characterization $(1)+(2)$ relies on the Gaussian behaviour of the Euclidean heat kernel for small times. On an arbitrary Riemannian $m$-manifold such a Gaussian behaviour need not hold in general. As was shown by Kuwae-Takahashi [14], a genuine geometric assumptions that actually implies such a heat kernel behaviour is: Geodesic completeness plus Ricci curvature bounded from below and a positive injectivity radius. In [14], the authors also show (actually in a much more general context than Riemannian manifolds) that one has the characterization $(1)+(2)$ (with $\mathrm{d}_{g}(x, y)$ and the volume measure $\mu_{g}$ replacing their Euclidean analogues). In particular, now one also has

$$
\mathrm{L}^{q}\left(M, \mathrm{~d} \mu_{g}\right) \subset \mathcal{K}(M, g) \text { with } q \text { as in }(2) .
$$

If one insists on an $\mathrm{L}^{q}$-Kato-criterion which is precisely of the form (4), seemingly there is not much to improve the geometric assumptions of Kuwae and Takahasi. On the other

\footnotetext{
${ }^{1}$ uniformly local $q$-integrability instead of globally $q$-integrability is sufficient
} 
hand, a positive injectivity radius assumption is very restrictive in applications, and furthermore, in view of the above essential self-adjointness result one might want to know if or under which assumptions one has

$$
\mathrm{L}_{\text {loc }}^{q}(M) \subset \mathcal{K}_{\text {loc }}(M, g) \text { with } q \text { as in }(2)
$$

In this context, we prove the following results in this paper:

- Given an appropriate continuous control function $I: M \rightarrow(0, \infty)$ for the heat kernel $p_{g}(t, x, y)$ (cf. Definition 2.3) one always has an global inclusion of the form (cf. Theorem 2.5)

$$
\mathrm{L}^{q}\left(M, I \mathrm{~d} \mu_{g}\right) \subset \mathcal{K}(M, g) \text {, with } q \text { as in (2). }
$$

- Using a parabolic $\mathrm{L}^{1}$-mean value inequality it is possible to prove a generally valid heat kernel estimate of the form

$$
\sup _{y \in M} p_{g}(t, x, y) \leq C \min \left(t, R(x)^{2}\right)^{-m / 2} \quad t>0, x \in M
$$

where $C>0$ is a universal constant, and $R=R_{g}: M \rightarrow(0, \infty)$ is a continuous function; in particular, $I=R^{-m}$ is a control function as above and, as $R$ is continuous, one always has (5) on every Riemannian manifold; this also implies that one can replace $\mathcal{K}_{\text {loc }}(M, g)$ with $\mathrm{L}_{\text {loc }}^{q}(M)$ in the above essential self-adjointness result IV) (cf. Corollary 2.13).

- If $(M, g)$ is geodesically complete with Ricci curvature bounded from below (without any additional assumption on the injectivity radiius), then one can pick the geometric control function $I_{g}$ given by the inverse volume function $1 / \mu(B(x, 1))$ with $B(x, 1)$ the open geodesic ball around $x$ with radius 1 (cf. Example 2.7), ending up with a global criterion of the form

$$
\mathrm{L}^{q}\left(M, I_{g} \mathrm{~d} \mu_{g}\right) \subset \mathcal{K}(M, g) \text {, with } q \text { as in (2). }
$$

We believe that these results close some rather long standing gaps to the Euclidean case in the context of $\mathrm{L}^{q}$-criteria for Kato functions on Riemannian manifolds that need not have a bounded geometry.

Another well-known Euclidean result is the following: Given a surjective linear map $T: \mathbb{R}^{m} \rightarrow \mathbb{R}^{m^{\prime}}$, one has $w \circ T \in \mathcal{K}\left(\mathbb{R}^{m}\right)$ for all $w \in \mathcal{K}\left(\mathbb{R}^{m^{\prime}}\right)$. This result is particularly important in the context of many-body quantum mechanics (cf. Example 2.15). Using an entirely probabilistic result by D. Elworthy [3] on the projections of Brownian motions, we found the following geometric variant of the latter Euclidean fact:

- Given a smooth minimal Riemannian submersion $\pi:(M, g) \rightarrow\left(M^{\prime}, g^{\prime}\right)$, one has $\pi^{*}\left[\mathcal{K}\left(M^{\prime}, g^{\prime}\right)\right] \subset \mathcal{K}(M, g)$ (cf. Theorem 2.14).

As, by what we have explained above, it is a rather tricky business to check the Kato property on noncompact manifolds, we believe that the latter result is particularly important for the construction of Kato functions in a noncompact curved setting.

Acknowledgements: This research has been supported by the SFB 647: Raum-ZeitMaterie. I would like to thank the referee for very precise and useful remarks. 


\section{MAIN RESUltS}

2.1. Definitions and $\mathrm{L}^{q}$-criteria for the Kato class. Let $M$ be a smooth connected manifold of dimension $m$. Given a smooth Riemannian metric $g$ on $M$, we denote with $B_{g}(x, r)$ the open geodesic balls and with $\mu_{g}(x, r):=\mu_{g}\left(B_{g}(x, r)\right)$ the volume function. The $\mathrm{L}^{q}$-spaces corresponding to $\mu_{g}$ will be denoted with $\mathrm{L}^{q}(M, g):=\mathrm{L}^{q}\left(M, \mathrm{~d} \mu_{g}\right)$. The minimal nonnegative heat kernel on $(M, g)$ is denoted with

$$
p_{g}(t, x, y), \quad(t, x, y) \in(0, \infty) \times M \times M .
$$

It is jointly smooth in $(t, x, y)$, and $p_{g}(\bullet, \bullet, y)$ is uniquely determined as the pointwise minimal function $u:(0, \infty) \times M \rightarrow[0, \infty)$ which satisfies

$$
(1 / 2) \Delta_{g} u(t, x)=\partial_{t} u(t, x), \lim _{t \rightarrow 0+} u(t, \bullet)=\delta_{y},
$$

where $\Delta_{g}=\mathrm{d}^{g} \mathrm{~d}$ denotes the negative definite Laplace-Beltrami operator.

Remark 2.1. Equivalently $[6], p_{g}(t, x, \bullet)$ is uniquely determined by

$$
p_{g}(t, x, y)=\mathrm{e}^{(t / 2) \Delta_{g}}(x, y)
$$

where here and in the sequel, by the usual abuse of notation, $(-1 / 2) \Delta_{g} \geq 0$ denotes the Friedrichs realization of the Laplace-Beltrami operator in the complex Hilbert space $\mathrm{L}^{2}(M, g)$. In particular, the symmetry $p_{g}(t, x, y)=p_{g}(t, y, x)$ is obvious from this point of view.

One always has

$$
\int p_{g}(t, x, y) \mathrm{d} \mu(y) \leq 1 \text { for all } t>0, x \in M
$$

and the Chapman-Kolmogorov identity

$$
\int p_{g}(t, x, z) p_{g}(s, z, y) \mathrm{d} \mu_{g}(z)=p_{g}(t+s, x, y) \quad \text { for all }(t, s) \in(0, \infty)^{2},(x, y) \in M^{2} .
$$

If one has equality in $(7)$ for some/all $(t, x)$, then $(M, g)$ is called stochastically complete. Under our standing assumption of connectedness, one always has $p_{g}(t, x, y)>0$.

It will be convenient to denote with $\mathscr{M}(M)$ the space of all smooth Riemannian metrics on $M$.

Definition 2.2. The Kato class $\mathcal{K}(M, g)$ of $g \in \mathscr{M}(M)$ is defined to be space of all Borel functions $w: M \rightarrow \mathbb{R}$, such that

$$
\lim _{t \rightarrow 0+} \sup _{x \in M} \int_{0}^{t} \int_{M} p_{g}(s, x, y)|w(y)| \mathrm{d} \mu_{g}(y) \mathrm{d} s=0,
$$

and the local Kato class $\mathcal{K}_{\text {loc }}(M, g)$ is given by all Borel $w: M \rightarrow \mathbb{R}$, such that $1_{K} w \in$ $\mathcal{K}(M, g)$ for any compact $K \subset M$. 
Both $\mathcal{K}(M, g)$ and $\mathcal{K}_{\text {loc }}(M, g)$ are linear spaces, and in view of

$$
\int p_{g}(t, x, y) \mathrm{d} \mu(y) \leq 1
$$

we always have the trivial inclusion $\mathrm{L}^{\infty}(M, g) \subset \mathcal{K}(M, g)$, noting that in fact $\mathrm{L}^{\infty}(M, g)$ does not depend on a particular choice of $g$. Using futher that $p_{g}(t, x, y)>0$ is continuous in $(t, x, y)$ it also follows easily that $\mathcal{K}_{\text {loc }}(M, g) \subset \mathrm{L}_{\text {loc }}^{1}(M)$ (cf. [10]).

Let us continue with (weighted) $\mathrm{L}^{q}$-criteria for the Kato class. To this end, we propose:

Definition 2.3 (Control data). a) An ordered pair $(I, \tilde{I})$ given by

- a continuous function $I: M \rightarrow(0, \infty)$

- a continuous function $\tilde{I}:(0,1] \rightarrow(0, \infty)$ such that for all $q \geq 1$ in case $m=1$, and all $q>m / 2$ in case $m \geq 2$, one has

$$
\int_{0}^{1} \tilde{I}(s)^{1 / q} \mathrm{~d} s<\infty
$$

is called a Kato control pair for $g \in \mathscr{M}(M)$, if for every $x \in M$ and every $0<t \leq 1$ one has

$$
\sup _{y \in M} p_{g}(t, x, y) \leq I(x) \tilde{I}(t)
$$

b) An ordered pair $(R, a)$ given by a continuous bounded function $R: M \rightarrow(0, \infty)$ and a number $a>0$ is called a Faber-Krahn control pair for $g \in \mathscr{M}(M)$, if for all $x \in M$, the ball $B_{g}(x, R(x))$ is relatively compact and if for every open $U \subset B_{g}(x, R(x))$ one has the Faber-Krahn type inequality

$$
\min \sigma\left(H_{\left.g\right|_{U}}\right) \geq a \mu_{g}(U)^{-\frac{2}{m}} .
$$

The following notation will be convenient in the sequel:

Definition 2.4. Given a Borel function $\Psi \geq 0$ on $M$ and $q \in[1, \infty)$, we denote with $\mathrm{L}^{q}(M, g, \Psi)$ the $\mathrm{L}^{q}$-space on $M$ with respect to the Borel measure $\Psi \mathrm{d} \mu_{g}$.

The importance of Kato control pairs stems from the following observation:

Theorem 2.5. For all

- $g \in \mathscr{M}(M)$,

- Kato control pairs $(I, \tilde{I})$ for $g$,

- $1 \leq q<\infty$ such that $q \geq 1$ if $m=1$, and $q>m / 2$ if $m \geq 2$,

- Borel functions $w: M \rightarrow \mathbb{R}$,

- $x \in M$, and all $0<s \leq 1$,

one has the bound

$$
\int p_{g}(s, x, y)|w(y)| \mathrm{d} \mu_{g}(y) \leq \tilde{I}(s)^{\frac{1}{q}}\left(\int|w(y)|^{q} I(y) \mathrm{d} \mu_{g}(y)\right)^{\frac{1}{q}} .
$$

In particular, for every $g \in \mathscr{M}(M)$, and for every choice of $q$ and $I$ as above one has

$$
\mathrm{L}^{q}(M, g, I) \subset \mathcal{K}(M, g) .
$$


Proof. Let us record the inequality

$$
\sup _{x \in M} p_{g}(s, x, y) \leq I(y) \tilde{I}(s)
$$

for $s \leq 1$, which follows from $p_{g}(s, x, y)=p_{g}(s, y, x)$. It is sufficient to show (10). In order to derive the latter note first that the case $q=1$ (which is only allowed for $m=1$ ) is obvious from (12), so assume $q>1$. Here the idea is to bound $\int p_{g}(s, x, y)|w(y)| \mathrm{d} \mu_{g}(y)$ is to factor the heat kernel appropriately: Indeed, with $1 / q+1 / q^{*}=1$, Hölder's inequality, and (21) we can estimate as follows,

$$
\begin{aligned}
& \int p_{g}(s, x, y)|w(y)| \mathrm{d} \mu_{g}(y)=\int p_{g}(s, x, y)^{\frac{1}{q^{*}}} p_{g}(s, x, y)^{1-\frac{1}{q^{*}}}|w(y)| \mathrm{d} \mu_{g}(y) \\
& \leq\left(\int p_{g}(s, x, y) \mathrm{d} \mu_{g}(y)\right)^{\frac{1}{q^{*}}}\left(\int|w(y)|^{q} p_{g}(s, x, y) \mathrm{d} \mu_{g}(y)\right)^{\frac{1}{q}} \\
& \leq\left(\int|w(y)|^{q} \tilde{I}(s) I(y) \mathrm{d} \mu_{g}(y)\right)^{\frac{1}{q}} \leq \tilde{I}(s)^{\frac{1}{q}}\left(\int|w(y)|^{q} I(y) \mathrm{d} \mu_{g}(y)\right)^{\frac{1}{q}} .
\end{aligned}
$$

This completes the proof.

The following examples provide some typical examples of the above notions. In particular, it shows that every smooth Riemannian manifold admits a Faber-Krahn control pair.

Example 2.6. 1. Given $g \in \mathscr{M}(M)$, assume that there exists a constant $C>0$ with

$$
\sup _{x \in M} p_{g}(t, x, x) \leq C t^{-m / 2} \text { for all } 0<t \leq 1
$$

Then using the inequality

$$
p_{g}(t, x, y) \leq \sqrt{p_{g}(t, x, x)} \sqrt{p_{g}(t, y, y)}
$$

which follows easily from the above general properties of the minimal heat kernel, we find that $(I(x), \tilde{I}(t)):=\left(C, t^{-m / 2}\right)$ is a Kato control pair for $g$, which is constant in its first slot. 2. For an arbitrary $g \in \mathscr{M}(M)$, given $x \in M, b>1$, define a Euclidean radius $r_{\text {Eucl }, g}(x, b)$ of accuracy $b$ to be the supremum of all $r>0$ such that $B_{g}(x, r)$ is relatively compact and admits a chart with respect to which one has one has the following inequality for all $y \in B_{g}(x, r)$,

$$
\frac{1}{b}\left(\delta_{i j}\right) \leq\left(g_{i j}(y)\right) \leq b\left(\delta_{i j}\right) \text { as symmetric bilinear forms. }
$$

Then for all $\epsilon_{1}>0, \epsilon_{2}>1$ the function defined by $R(x):=\min \left(r_{\text {Eucl }, g}(x, b), \epsilon_{1}\right) / \epsilon_{2}$ fits into a Faber-Krahn control pair for $g$. In this case, $R$ is even $1 / \epsilon_{2}$-Lipschitz with respect to $\mathrm{d}_{g}$.

Under curvature bounds, one can construct very explicit Kato control pairs. The following example treats manifolds with a Ricci curvature bounded below by a constant in such a context: 
Example 2.7. Assume $m \geq 2$. For every $\kappa \geq 0$ there exist constants $C_{j}=C_{j}(\kappa, m)$ which only depend on $\kappa, m$, such that for all geodesically complete $g \in \mathscr{M}(M)$ with $\mathrm{Ric}_{g} \geq-\kappa$, and all $t>0, x, y \in M$ one has the well-known Li-Yau estimate

$$
p_{g}(t, x, y) \leq C_{1} \mu_{g}\left(B_{g}(x, \sqrt{t})\right)^{-1} \exp \left(-\frac{\mathrm{d}_{g}(x, y)^{2}}{C_{2} t}+C_{3} t\right) .
$$

In addition one has the following volume 'doubling': For every $0<s^{\prime} \leq s, x \in M$, one has

$$
\mu_{g}(B(x, s)) \leq \mu_{g}\left(B_{g}\left(x, s^{\prime}\right)\right)\left(s / s^{\prime}\right)^{m} \exp (\sqrt{(m-1) \kappa} s) .
$$

Thus for $t \leq 1$, we have

$$
\mu_{g}\left(B_{g}(x, \sqrt{t})\right)^{-1} \leq C_{4} \mu_{g}\left(B_{g}(x, 1)\right)^{-1} t^{m / 2},
$$

and we have derived the heat kernel control pair given by

$$
I(x):=C_{5} \mu_{g}\left(B_{g}(x, 1)\right)^{-1}, \quad \tilde{I}(t):=t^{m / 2},
$$

where $C_{4}=C_{4}(\kappa, m)>0, C_{5}=C_{5}(\kappa, m)>0$.

We immediately obtain the following new result:

Corollary 2.8. Assume $m=\operatorname{dim}(M) \geq 2$. Then given a geodesically complete $g \in \mathscr{M}(M)$ with $\operatorname{Ric}_{g} \geq-\kappa$ for some $\kappa>0$, it follows that for every $m / 2<q<\infty$ one has

$$
\mathrm{L}^{q}\left(M, g, \mu_{g}(\bullet, 1)^{-1}\right) \subset \mathcal{K}(M, g) .
$$

More precisely, for every $\kappa>0$ there exists a constant $C=C(m, \kappa)>0$, such that for all

- geodesically complete $g \in \mathscr{M}(M)$ with $\mathrm{Ric}_{g} \geq-\kappa$,

- $m / 2<q<\infty$,

- Borel functions $w: M \rightarrow \mathbb{R}$,

- $0<s \leq 1, x \in M$,

one has the inequality

$$
\int p_{g}(s, x, y)|w(y)| \mathrm{d} \mu_{g}(y) \leq C^{1 / q} s^{-m /(2 q)}\left(\int|w(x)|^{q} \mu_{g}(x, 1)^{-1} \mathrm{~d} \mu_{g}(x)\right)^{\frac{1}{q}} .
$$

Let us return to the case of general manifolds again. Part b) of the following result shows that every Faber-Krahn control pair canonically induces a Kato control pair:

Theorem 2.9. There exists a constant $C=C(m)>0$, which only depends on $m$, such that for every $g \in \mathscr{M}(M)$, every Faber-Krahn control pair $(R, a)$ for $g$, and every $t>0$, $x \in M$, one has

$$
\sup _{y \in M} p_{g}(t, x, y) \leq C a^{-m / 2} \min \left(t, R(x)^{2}\right)^{-m / 2} .
$$

In particular, there exists a constant $C=C(m)>0$, such that for every $g \in \mathscr{M}(M)$ and every Faber-Krahn control pair $(R, a)$ for $g$, the assignment

$$
I(x):=C a^{-m / 2} R(x)^{-m}, \quad \tilde{I}(t):=\left(t^{-m / 2}\left(\sup R^{m}\right)+1\right)
$$

defines a Kato control pair for $g$. 
Once one has (14), the fact that (15) defines a Kato control pair follows from

$$
\min \left(t, R(x)^{2}\right)^{-m / 2} \leq t^{-m / 2}+R(x)^{-m} \leq R(x)^{-m}\left(t^{-m / 2}\left(\sup R^{m}\right)+1\right) .
$$

The proof of (14) requires a parabolic $L^{1}$-mean value inequality (MVI), the latter of which has been stated in [5] without proof. There it is also pointed out that the parabolic $\mathrm{L}^{1}$-MVI can be deduced from its well-known $\mathrm{L}^{2}$-analogue by using methods from [16]. As it does not cause much extra work and as it could be useful elsewhere, we give a detailed proof of a parabolic $\mathrm{L}^{q}$-mean value inequality $(1 \leq q \leq 2)$, for the convenience of the reader:

Proposition 2.10 (Parabolic $\left.\mathrm{L}^{q} \mathrm{M} \mathrm{MVI}\right)$. There exists a constant $C=C(m)>0$, which only depends on $m$, with the following property:

- for all $g \in \mathscr{M}(M), x \in M, r>0$ with $B_{g}(x, r)$ relatively compact and admitting a constant $a>0$ such that for every open $U \subset B_{g}(x, r)$ one has the Faber-Krahn inequality (9),

- for all $\tau \in\left(0, r^{2}\right], t \geq \tau$,

- for all nonnegative solutions $u$ of the g-heat equation

$$
\partial_{t} u=(1 / 2) \Delta_{g} u \quad \text { in }(t-\tau, t] \times B_{g}(x, \sqrt{\tau}),
$$

- for all $q \in[1,2]$

one has the bound

$$
u(t, x)^{q} \leq \frac{C}{a^{\frac{m}{2}} \tau^{1+\frac{m}{2}}} \int_{t-\tau}^{t} \int_{B_{g}(x, r)} u(s, y)^{q} \mathrm{~d} \mu_{g}(y) \mathrm{d} s .
$$

Proof. Applying Theorem 15.1 in [6] (a slightly different formulation of an $\mathrm{L}^{2}$-MVI) to the radius $\sqrt{\tau}$ and to the solution

$$
(0, \tau] \times B_{g}(x, \sqrt{\tau}) \ni(s, y) \longmapsto u(t-\tau+s, y) \in[0, \infty)
$$

of the $g$-heat equation in $(0, \tau] \times B_{g}(x, \sqrt{\tau})$, immediately implies the $\mathrm{L}^{2}$-MVI

$$
u(t, x)^{2} \leq \frac{C a^{-\frac{m}{2}}}{\tau^{1+\frac{m}{2}}} \int_{t-\tau}^{t} \int_{B_{g}(x, \sqrt{\tau})} u(s, y)^{2} \mathrm{~d} \mu_{g}(y) \mathrm{d} s .
$$

From here on we apply a modified $\mathrm{L}^{q}$-to- $\mathrm{L}^{1}$ version of the parabolic $\mathrm{L}^{2}$-to- $\mathrm{L}^{1}$ reduction machinery from pp. $1269 / 1270$ in [16]. So let $1 \leq q<2$. Setting

$$
D:=C a^{-\frac{m}{2}} 4^{1+m / 2},
$$

and applying (17) with $\tau$ replaced with $\tau / 4$ implies

$$
u(t, x)^{2} \leq D \tau^{-(1+m / 2)} \int_{t-\tau / 4}^{t} \int_{B_{g}(x, \sqrt{\tau} / 2)} u(s, y)^{2} \mathrm{~d} \mu_{g}(y) \mathrm{d} s,
$$

so that setting

$$
Q:=\tau^{-(1+m / 2)} \int_{t-\tau}^{t} \int_{B_{g}(x, \sqrt{\tau})} u(s, y)^{q} \mathrm{~d} \mu_{g}(y) \mathrm{d} s
$$


and for every $k \in \mathbb{N}$,

$$
S_{k}:=\sup _{\left[t-\tau \sum_{i=1}^{k} 4^{-i}, t\right] \times B_{g}\left(x, \sqrt{\tau} \sum_{i=1}^{k} 2^{-i}\right)} u^{2-q}
$$

we immediately get

$$
u(t, x)^{2} \leq D Q S_{1} .
$$

Let us next prove that for all $k$ one has

$$
S_{k} \leq\left(D Q S_{k+1}\right)^{\alpha}
$$

where

$$
\alpha:=(2-q) / 2 \text {. }
$$

To see the latter, pick

$$
(s, y) \in\left[t-\tau \sum_{i=1}^{k} 4^{-i}, t\right] \times B_{g}\left(x, \sqrt{\tau} \sum_{i=1}^{k} 2^{-i}\right)
$$

with $u(s, y)^{2-q}=S_{k}$. Applying now (17) with $t$ replaced with $s$, and $\tau$ replaced with $\tau / 4^{k+1}$ and using

$$
\left[t-\tau \sum_{i=1}^{k+1} 4^{-i}, t\right] \times B_{g}\left(x, \sqrt{\tau} \sum_{i=1}^{k+1} 2^{-i}\right) \supset\left[s-\tau / 4^{k+1}, s\right] \times B_{g}\left(y, \sqrt{\tau} / 2^{k+1}\right)
$$

to estimate the resulting space-time integral, we get

$$
u(s, y)^{2} \leq D Q S_{k+1},
$$

which implies (19). We claim that for all $k$ one has

$$
u(t, x)^{2} \leq D^{\sum_{i=1}^{k} \beta^{-i+1}} Q^{\sum_{i=1}^{k} \beta^{-i+1}} S_{k}^{\frac{1}{\beta^{k-1}}} .
$$

where

$$
\beta:=1 / \alpha \text {. }
$$

The proof is by induction on $k$ : The case $k=1$ has already been shown in (18). Given the statement for $k$, we have using (19),

$$
\begin{aligned}
& u(t, x)^{2} \leq D^{\sum_{i=1}^{k} \beta^{-i+1}} Q^{\sum_{i=1}^{k} \beta^{-i+1}} S_{k}^{\frac{1}{\beta^{k-1}}} \leq D^{\sum_{i=1}^{k} \beta^{-i+1}} Q^{\sum_{i=1}^{k} \beta^{-i+1}} D^{1 / \beta^{k}} Q^{1 / \beta^{k}} S_{k+1}^{1 / \beta^{k}} \\
& =D^{\sum_{i=1}^{k+1} \beta^{-i+1}} Q^{\sum_{i=1}^{k+1} \beta^{-i+1}} S_{k+1}^{\frac{1}{\beta^{k}}},
\end{aligned}
$$


which completes the proof of $(20)$. As $\left(S_{k}\right)_{k}$ is a bounded sequence ${ }^{2}$ we now get from letting $k \rightarrow \infty$ in $(20)$ the bound

$$
u(t, x)^{2} \leq D^{\sum_{i=0}^{\infty} \beta^{-i}} Q^{\sum_{i=0}^{\infty} \beta^{-i}} \lim _{k \rightarrow \infty} S_{k}^{\frac{1}{\beta^{k-1}}}=(D Q)^{\beta /(\beta-1)} .
$$

Recalling that

$$
\beta /(\beta-1)=1-(2-q) / 2=2 / q,
$$

the latter bound completes the proof of the $\mathrm{L}^{q}-\mathrm{MVI}$, in view of $\tau \leq r^{2}$.

Being equipped with Proposition 2.10, we can now give the

Proof of Theorem 2.9. As we have already stated, it remains to prove (14). To this end, fix arbitrary $t>0, x, y \in M$. As

$$
(s, z) \mapsto u(s, z):=p_{g}(s, z, y)
$$

is a nonnegative solution of the $g$-heat equation on $(0, \infty) \times M$, an application of Proposition 2.10 with $r:=R(x)$ immediately implies

$$
p_{g}(t, x, y) \leq \frac{C a^{-\frac{m}{2}}}{\tau^{1+\frac{m}{2}}} \int_{t-\tau}^{t} \int_{M} p_{g}(s, z, y) \mathrm{d} \mu_{g}(z) \mathrm{d} s,
$$

for all $\tau \in\left(0, R(x)^{2}\right]$. As we have

$$
\int_{M} p_{g}\left(s, z, y^{\prime}\right) \mathrm{d} \mu_{g}(z)=\int_{M} p_{g}\left(s, y^{\prime}, z\right) \mathrm{d} \mu_{g}(z) \leq 1 \quad \text { for all }\left(s, y^{\prime}\right) \in(0, \infty) \times M,
$$

we arrive at $p_{g}(t, x, y) \leq C a^{-\frac{m}{2}} \tau^{-\frac{m}{2}}$, which proves the result, upon taking $\tau:=\min \left(R(x)^{2}, t\right)$.

In view of Example 2.6 and Theorem 2.5 we now immediately get the following result, which we believe is much more subtle than it looks at first sight:

Corollary 2.11. Every $g \in \mathscr{M}(M)$ admits a Kato control pair. In particular, for every $1 \leq q<\infty$ such that $q \geq 1$ if $m=1$, and $q>m / 2$ if $m \geq 2$, one has $\mathrm{L}_{\text {loc }}^{q}(M) \subset \mathcal{K}_{\text {loc }}(M, g)$.

Proof. By Example 2.6 and Theorem 2.9 we can pick a Kato control pair $(I, \tilde{I})$ for $g$. Given a compact $K \subset M$ and $w \in \mathrm{L}_{\text {loc }}^{q}(M)$ one has

$$
\int_{K}|w|^{q} I \mathrm{~d} \mu_{g} \leq\left(\max _{K} I\right) \int_{K}|w|^{q} \mathrm{~d} \mu_{g}<\infty,
$$

as $I$ is continuous, thus $1_{K} w \in \mathcal{K}(M)$ by Corollary to 2.5.

We believe that Corollary 2.11 suggests the

\footnotetext{
${ }^{2}$ for example, we have (estimating the sums with geometric series)

$$
S_{k}=\sup _{\left[t-\tau \sum_{i=1}^{k} 4^{-i}, t\right] \times B_{g}\left(x, \sqrt{\tau} \sum_{i=1}^{k} 2^{-i}\right)} u^{2-q} \leq \sup _{\left[t-\frac{3}{4} \tau, t\right] \times B_{g}(x, \sqrt{\tau})} u^{2-q}<\infty .
$$
}


Open problem 2.12. Is there a (large) class $\tilde{\mathscr{M}}(M) \subset \mathscr{M}(M)$ such that for all $g, h \in$ $\tilde{\mathscr{M}}(M)$ one has $\mathcal{K}_{\text {loc }}(M, g)=\mathcal{K}_{\text {loc }}(M, h)$ ?

A systematic treatment of this problem probably requires a generally valid heat kernel bound as in (14) with a damping Gaussian factor, and a matching lower bound.

2.2. Essential self-adjointness. Using a result [7] on the essential-self-adjointness of Schrödinger operator with singular magnetic potentials, and locally Kato electric potentials, the Corollary 2.11 implies the following:

Corollary 2.13. Assume that

- $g \in \mathscr{M}(M)$ is geodesically complete,

- the real-valued 1-form ("the magnetic potential")

$\alpha \in \Gamma_{\mathrm{L}_{\mathrm{loc}}^{4}}\left(M, T^{*} M\right)$ has a weak gradient $\operatorname{grad}_{g}(\alpha) \in \mathrm{L}_{\mathrm{loc}}^{2}(M)$,

- $w \in \mathrm{L}_{\text {loc }}^{2}(M)$ is real-valued ("the electric potential") with $w \in \mathrm{L}_{\text {loc }}^{q}(M)$ for some $1 \leq q<\infty$, which satisfies $q \geq 1$ if $m=1$, and $q>m / 2$ if $m \geq 2$.

- the symmetric operator $H_{g}^{\alpha, w}$ in the complex Hilbert space $\mathrm{L}^{2}(M, g)$ given by

$$
H_{g}^{\alpha, w} \Psi=-\Delta_{g} \Psi-2 \sqrt{-1} g^{*}(\alpha, \mathrm{d} \Psi)+\left(\sqrt{-1} \operatorname{grad}_{g}(\alpha)+|\alpha|_{g^{*}}^{2}+w\right) \Psi, \quad \Psi \in \mathrm{C}_{\mathrm{c}}^{\infty}(M),
$$

is bounded from below.

Then $H_{g}^{\alpha, w}$ is essentially self-adjoint, in other words, $H_{g}^{\alpha, w}$ has precisely one self-adjoint extension.

Proof. If $(M, g)$ is geodesically complete, $\alpha$ is as above and $v \in \mathrm{L}_{\text {loc }}^{2}(M) \cap \mathcal{K}_{\text {loc }}(M, g)$ is real-valued such that $H_{g}^{\alpha, v}$ is bounded from below, then the essential self-adjointness of $H_{g}^{\alpha, v}$ has been shown in [7]. Now the result follows from Corollary 2.11.

Concerning the assumptions of Corollary 2.13: If $(M, g)$ is geodesically complete, $\alpha \in$ $\Gamma_{\mathrm{L}_{\text {loc }}^{4}}\left(M, T^{*} M\right)$ is real-valued with $\operatorname{grad}_{g}(\alpha) \in \mathrm{L}_{\text {loc }}^{2}(M)$, and $w \in \mathrm{L}_{\text {loc }}^{2}(M)$ is bounded from below, then it is reasonable to expect that $H_{g}^{\alpha, w}$ automatically is essentially self-adjoint without any further $\mathrm{L}_{\text {loc }}^{q}$-assumptions on $w$; indeed this is known on manifolds for smooth $\alpha$ 's [2], and in $\mathbb{R}^{m}$ for arbitrary $\alpha$ 's. However, such an assumption on the electric potential is almost never satisfied in quantum physics (where we typically have $w(x) \sim-|x|^{-1}$; cf: Example 2.15). The point of Corollary 2.13 is that it does not require semiboundedness on the electric potential, but only on the operator itself, with the small price of requiring an additional $\mathrm{L}_{\text {loc }}^{q}$-assumption if $m>3$. In particular, in the most important case $m=3$, these assumptions are satisfied if $w(x) \sim-|x|^{-1}$.

\subsection{Projecting Kato functions. In this section we prove:}

Theorem 2.14. Let $M^{\prime}$ be another smooth connected manifold, let $g \in \mathscr{M}(M), g^{\prime} \in$ $\mathscr{M}\left(M^{\prime}\right)$ and let $\pi:(M, g) \rightarrow\left(M^{\prime}, g^{\prime}\right)$ be a smooth surjective map such that 
- $\pi$ is a Riemannian submersion, that is, the vector bundle (iso)morphism

$$
\left.T \pi\right|_{\operatorname{ker}(T \pi)^{\perp g}}:\left(\operatorname{ker}(T \pi)^{\perp_{g}}, g\right) \longrightarrow\left(T M^{\prime}, g^{\prime}\right)
$$

is fiberwise orthogonal

- for all $y \in M^{\prime}$ the fiber $\pi^{-1}(y) \subset(M, g)$ is a minimal submanifold, that is, the g-mean curvature

$$
H^{\pi^{-1}(y), g} \in \Gamma_{\mathrm{C}^{\infty}}\left(\pi^{-1}(y), T^{*} \pi^{-1}(y) \odot T^{*} \pi^{-1}(y)\right)
$$

of the submanifold $\pi^{-1}(y)$ vanishes identically.

Then for all Borel $w: M^{\prime} \rightarrow \mathbb{R}, t>0, x \in M$, there is the bound

$$
\int_{M} p_{g}(t, x, y)|w(\pi(y))| \mathrm{d} \mu_{g}(y) \leq \int_{M^{\prime}} p_{g^{\prime}}(t, \pi(x), z)|w(z)| \mathrm{d} \mu_{g^{\prime}}(z) .
$$

In particular, for all $w \in \mathcal{K}\left(M^{\prime}, g^{\prime}\right)$ one has $w \circ \pi \in \mathcal{K}(M, g)$, and, furthermore, if $(M, g)$ is stochastically complete, then so is $\left(M^{\prime}, g^{\prime}\right)$.

Proof. Let us first remark that obviously it is enough to prove (22).

The bound (22) follows from a result by Elworthy (Theorem 10 E on p.256 in [3]), which states that under the given assumptions on $\pi$, Brownian motions on $(M, g)$ are projected to restrictions of Brownian motions on $\left(M^{\prime}, g^{\prime}\right)$. Since we have to deal with explosion times, the precise statement of the the latter result is a little technical which is why we recall some definitions:

Let $(N, h)$ be an arbitrary Riemannian manifold. Given a probability space $(\Omega, \mathscr{F}, \mathbb{P})$, a Brownian motion $X\left(x_{0}\right)$ on $(N, h)$ with starting point $x_{0} \in N$ is given by a pair $\left(X\left(x_{0}\right), \zeta\left(x_{0}\right)\right)$ which satisfies the following assumptions:

- $\zeta\left(x_{0}\right): \Omega \rightarrow[0, \infty]$ is measurable such that, $\mathbb{P}$-a.s., one has $\zeta\left(x_{0}\right)>0$ as well as

$$
1_{\left\{\zeta\left(x_{0}\right)<\infty\right\}} \leq 1_{\left\{\lim _{t \rightarrow \zeta\left(x_{0}\right)-} X_{t}\left(x_{0}\right)=\infty_{N}\right\}}
$$

where the limit $\lim _{t \rightarrow \zeta\left(x_{0}\right)-} X_{t}\left(x_{0}\right)=\infty_{N}$ is understood with respect to the (essentially uniquely determined) Alexandrov compactification $\left(N, \infty_{N}\right)$ of $N$

- $X\left(x_{0}\right)$ is a process

$$
X\left(x_{0}\right):\left[0, \zeta\left(x_{0}\right)\right) \times \Omega:=\{(t, \omega) \in[0, \infty) \times \Omega\} \longrightarrow N
$$

with continuous paths

- for all $l \in \mathbb{N}$, all bounded Borel functions $f: N \rightarrow \mathbb{R}^{l}$ and all $0<t_{1}<\cdots<t_{l}$, one has

$$
\begin{aligned}
& \mathbb{E}\left[1_{\left\{t_{l}<\zeta\left(x_{0}\right)\right\}} f_{1}\left(X_{t_{1}}\left(x_{0}\right)\right) \ldots f_{l}\left(X_{t_{l}}\left(x_{0}\right)\right)\right] \\
& =\int \ldots \int p_{h}\left(\delta_{0}, x_{0}, x_{1}\right) f_{1}\left(x_{1}\right) \cdots p_{h}\left(\delta_{n-1}, x_{n-1}, x_{n}\right) f_{n}\left(x_{n}\right) \mathrm{d} \mu_{h}\left(x_{1}\right) \cdots \mathrm{d} \mu_{h}\left(x_{n}\right),
\end{aligned}
$$

where $\delta_{j}:=t_{j+1}-t_{j}, t_{0}:=0$. 
Note that in view of (6), the assumption (23) implies $X_{0}(x)=x \mathbb{P}$-a.s., as it should be. Furthermore, in the above situation, we will simply write

$$
X(x):[0, \zeta(x)) \times \Omega \longrightarrow(N, h)
$$

to indicate that $X(x)$ is a Brownian motion on $(N, h)$ with starting point $x \in N$, and call a map (to be precise, a pair of maps $(X, \zeta)$ )

$$
X: N \times[0, \zeta) \times \Omega \longrightarrow(N, h)
$$

a Brownian family, if for all $x \in N$,

$$
X(x):[0, \zeta(x)) \times \Omega \longrightarrow(N, h)
$$

is a Brownian motion with starting point $x \in N$.

Returning now to the actual statement of the Theorem, given an arbitary $x \in M$, set $x^{\prime}:=\pi(x)$. We can now formulate Elworthy's result (cf. the proof of Theorem $10 \mathrm{E}$ on p.256 in [3]): There exists a complete probability space $(\Omega, \mathscr{F}, \mathbb{P})$, and families of Brownian motions

$$
X: M \times[0, \eta) \times \Omega \longrightarrow(M, g), Y: M^{\prime} \times[0, \zeta) \times \Omega \longrightarrow\left(M^{\prime}, g^{\prime}\right)
$$

such that

$$
\mathbb{P}\left\{\zeta\left(x^{\prime}\right) \geq \eta(x),\left.Y\left(x^{\prime}\right)\right|_{[0, \eta(x)) \times \Omega}=\pi(X(x))\right\}=1 .
$$

The reader may find results of this type for more general diffusions than Brownian motion in [17]. It follows that for all $t>0, x \in M$,

$$
\begin{aligned}
& \int_{M} p_{g}(t, x, y)|w(\pi(y))| \mathrm{d} \mu_{g}(y)=\mathbb{E}\left[1_{\{t<\eta(x)\}} \mid w\left(\pi\left(X_{t}(x)\right) \mid\right]=\mathbb{E}\left[1_{\{t<\eta(x)\}}\left|w\left(Y_{t}\left(x^{\prime}\right)\right)\right|\right]\right. \\
& \leq \mathbb{E}\left[1_{\left\{t<\zeta\left(x^{\prime}\right)\right\}}\left|w\left(Y_{t}\left(x^{\prime}\right)\right)\right|\right]=\int_{M^{\prime}} p_{g^{\prime}}\left(t, x^{\prime}, z\right)|w(z)| \mathrm{d} \mu_{g^{\prime}}(z),
\end{aligned}
$$

which proves everything.

Given $g \in \mathscr{M}(M), l \in \mathbb{N}$, if we equip the product manifold $M^{l}=M \times \cdots \times M(l$ times) with the product Riemannian structure $\otimes^{l} g=g \otimes \cdots \otimes g$ (l-times), the canonical projections

$$
\pi_{i j}:\left(M^{l}, \otimes{ }^{l} g\right) \longrightarrow(M \times M, g \otimes g), i \neq j=1, \ldots, l,
$$

satisfy the assumptions of the previous result. In particular,

$$
w \in \mathcal{K}(M \times M, g \otimes g) \Rightarrow w \circ \pi_{i j} \in \mathcal{K}\left(M^{l}, \otimes^{l} g\right) .
$$

Likewise, we have

$$
w \in \mathcal{K}(M, g) \Rightarrow w \circ \pi_{j} \in \mathcal{K}\left(M^{l}, \otimes^{l} g\right),
$$

where $\pi_{j}: M^{l} \rightarrow M$ denotes the projection onto the $j$-th variable. This is precisely the situation that arises in many-body quantum mechanics: 
Example 2.15. Given $g \in \mathscr{M}(M)$ on the 3-manifold $M$, assume that for some $C>0$ and all $t>0, x \in M$ one has $p_{g}(t, x, x) \leq C t^{-3 / 2}$. Then the Coulomb potential

$$
V_{g}(x, y):=\frac{1}{2} \int_{0}^{\infty} p_{g}(s, x, y) \mathrm{d} s
$$

is finite for all $x \neq y$, and using the Chapman-Kolomogorow identity one easily finds [9] $V_{g}(\bullet, y) \in \mathcal{K}(M, g)$ for all fixed $y \in M$. Likewise, using the product rule (cf. Theorem 9.11 in [6] and the remark thereafter)

$$
p_{g \otimes g}\left(t,(x, y),\left(x^{\prime}, y^{\prime}\right)\right)=p_{g}\left(t, x, x^{\prime}\right) p_{g}\left(t, y, y^{\prime}\right), \quad(x, y),\left(x^{\prime}, y^{\prime}\right) \in M \times M,
$$

it is easily checked that $V_{g} \in \mathcal{K}(M \times M, g \otimes g)$. It follows that for all $1 \leq l_{1}, l_{2} \in \mathbb{N}$, all $i=1, \ldots, l_{1}, j=1, \ldots, l_{2}, y_{1} \ldots, y_{l_{2}} \in M$ one has

$$
V_{g ; i j ; y_{j}}:=-V_{g}\left(\pi_{i}, y_{j}\right) \in \mathcal{K}\left(M^{l}, \otimes^{l} g\right) .
$$

Likewise, for all $i, j=1, \ldots, l_{1}$ with $i<j$ the potentials

$$
W_{g ; i j}:=V_{g}\left(\pi_{i j}\right) \in \mathcal{K}\left(M^{l}, \otimes^{l} g\right)
$$

are Kato. Up to positive constants, the self-adjoint realizations of

$$
H\left(g ; y_{1}, \ldots, y_{l_{2}}\right):=-(1 / 2) \Delta_{\otimes l}+\sum_{i=1}^{l_{1}} \sum_{j=1}^{l^{2}} V_{g ; i j ; y_{j}}+\sum_{i, j=1, \ldots, l_{1}, i<j} W_{g ; i j}
$$

in the complex Hilbert space $\mathrm{L}^{2}\left(M^{l_{1}}, \otimes^{l_{1}} g\right)$ describe nonrelativistically the energy of $l_{1}$ electrons that live on $M$ under the influence of $l_{2}$ nuclei, where the $j$-th nucleus is considered to be fixed in $y_{j}$. Here, $V_{g ; i j ; x_{j}}$ is the interaction of the $i$-th electron with the $j$-th nucleus (which is thus attractive), and $W_{g ; i j}$ the interaction of the $i$-th electron with the $j$-th electron (which is thus repulsive).

\section{Appendix A. An $\mathrm{L}^{q}(M, g) \rightarrow \mathrm{L}^{q}(M, g)$-BOUnd FOR SCHRÖDINGER OpERATors With KATO POTENTIALS}

In this section we give a simple proof of:

Proposition A.1. For every

- $g \in \mathscr{M}(M)$

- Borel function $w: M \rightarrow \mathbb{R}$ which can be decomposed as $w=w_{+}-w_{-}$into Borel functions $w_{ \pm}: M \rightarrow[0, \infty)$ with $w_{+} \in \mathrm{L}_{\text {loc }}^{1}(M), w_{-} \in \mathcal{K}(M, g)$,

- $\delta>1$

\footnotetext{
${ }^{3}$ To be precise, we should actually consider $H\left(g, y_{1}, \ldots, y_{l_{2}}\right)$ on the closed subspace $\wedge^{l_{1}} \mathrm{~L}^{2}(M, g)$ of $\mathrm{L}^{2}\left(M^{l_{1}}, \otimes^{l_{1}} g\right)$; this is essential for questions like stability of matter [15]; similar stability results have been recently also obtained by the author and Enciso on the Riemannan 3-manifolds under consideration $[9,4]$
} 
there exists a constant $0 \leq C\left(w_{-}, \delta, g\right)<\infty$, such that for all $t \geq 0, q \in[1, \infty]$ one has

$$
\left\|\mathrm{e}^{-t H_{g}^{w}}\right\|_{\mathrm{L}^{q}(M, g) \rightarrow \mathrm{L}^{q}(M, g)} \leq \delta \mathrm{e}^{t C\left(w_{-}, \delta, g\right)} .
$$

Above, the Schrödinger operator $H_{g}^{w}=-(1 / 2) \Delta_{g}+w$ is well-defined as the self-adjoint operator in $\mathrm{L}^{2}(M, g)$ which corresponds to the closed semibounded (from below) densely defined symmetric sesqui-linear form

$$
Q_{g}^{w}\left(f_{1}, f_{2}\right):=\frac{1}{2} \int g^{*}\left(\mathrm{~d} f_{1}, \mathrm{~d} f_{2}\right) \mathrm{d} \mu_{g}+\int \overline{w f_{1}} f_{2} \mathrm{~d} \mu_{g}
$$

with domain of definition $\operatorname{Dom}\left(Q_{g}^{w}\right)=\mathrm{W}_{0}^{1,2}(M, g) \cap \mathrm{L}^{2}\left(M, g, w_{+}\right)$.

We will use Riesz-Thorin's Theorem for the proof:

Theorem A.2 (Riesz-Thorin). Let $\left(X, \mu_{X}\right)$ and $\left(Y, \mu_{Y}\right)$ be sigma-finite measure spaces, let $a_{0}, a_{1}, b_{0}, b_{1} \in[1, \infty]$, and assume that

$$
T: \mathrm{L}^{a_{0}}\left(X, \mu_{X}\right) \cap \mathrm{L}^{a_{1}}\left(X, \mu_{X}\right) \longrightarrow \mathrm{L}^{b_{0}}\left(Y, \mu_{Y}\right) \cap \mathrm{L}^{b_{1}}\left(Y, \mu_{Y}\right)
$$

is a complex linear map. Assume further that there are numbers $C_{0}, C_{1}>0$ such that for all $f \in \mathrm{L}^{a_{0}}\left(X, \mu_{X}\right) \cap \mathrm{L}^{a_{1}}\left(X, \mu_{X}\right)$ one has

$$
\|T f\|_{\mathrm{L}^{b_{0}\left(Y, \mu_{Y}\right)}} \leq C_{0}\|f\|_{\mathrm{L}^{a_{0}\left(X, \mu_{X}\right)}},\|T f\|_{\mathrm{L}^{b_{1}\left(Y, \mu_{Y}\right)}} \leq C_{1}\|f\|_{\mathrm{L}^{a_{1}\left(X, \mu_{X}\right)}} .
$$

Then for any $r \in[0,1]$, there is a unique bounded extension

$$
T_{a_{r}, b_{r}} \in \mathscr{L}\left(\mathrm{L}^{a_{r}}\left(X, \mu_{X}\right), \mathrm{L}^{b_{r}}\left(Y, \mu_{Y}\right)\right)
$$

of $T$, which satisfies

$$
\left\|T_{a_{r}, b_{r}}\right\|_{\mathrm{L}^{a_{r}}\left(X, \mu_{X}\right) \rightarrow \mathrm{L}^{b_{r}\left(Y, \mu_{Y}\right)}} \leq C_{0}^{1-r} C_{1}^{r}, \quad \text { where } \frac{1}{a_{r}}:=\frac{1-r}{a_{0}}+\frac{r}{a_{1}}, \frac{1}{b_{r}}:=\frac{1-r}{b_{0}}+\frac{r}{b_{1}},
$$

with the usual conventions $1 / \infty:=0,1 / 0:=\infty$.

Proof of Proposition A.1. Let us record the Feynman-Kac formula

where

$$
\mathrm{e}^{-t H_{g}^{w}} f(x)=\mathbb{E}\left[1_{\{t<\zeta(x)\}} \mathrm{e}^{-\int_{0}^{t} w\left(X_{s}(x)\right) \mathrm{d} s} f\left(X_{t}(x)\right)\right], f \in \mathrm{L}^{2}(M, g),
$$

$$
X: M \times[0, \zeta) \times \Omega \longrightarrow(M, g)
$$

is a family of Brownian motions on $(M, g)$. We extend $\mathrm{e}^{-t H_{g}^{w}}$ to $\mathrm{L}^{q}(M, g)$ by means of the rhs of the latter formula. Clearly, with this convention, we have

$$
\left|\mathrm{e}^{-t H_{g}^{w}} f(x)\right| \leq \mathrm{e}^{-t H_{g}^{-w_{-}}}|f|(x)
$$

and it remains to estimate the operator norms of $\mathrm{e}^{-t H_{g}^{-w_{-}}}$in each case.

By an adoption of a standard argument [1] that relies on the Markoff property of $B$ and the Kato property of $w_{-}$, one finds the following exponential estimate [8]: For every $\delta>1$ there exists a finite $C\left(w_{-}, \delta, g\right) \geq 0$ such that for all $t \geq 0$ one has

$$
C\left(w_{-}, t, g\right):=\sup _{x \in M} \mathbb{E}\left[1_{\{t<\zeta(x)\}} \mathrm{e}^{-\int_{0}^{t} w\left(X_{s}(x)\right) \mathrm{d} s}\right] \leq \delta \mathrm{e}^{t C\left(w_{-}, \delta, g\right)} .
$$


Coming to the proof of the actual statement of the proposition, note first that the case $q=\infty$ now follows immediately by what we have said above.

For the case $q=1$, let $h \in \mathrm{L}^{q}(M, g)$, and let $\bigcup_{n} K_{n}=M$ be a relatively compact exhaustion of $M$. Then we have

$$
\int\left|\mathrm{e}^{-t H_{g}^{-w_{-}}} h\right| \cdot 1_{K_{n}} \mathrm{~d} \mu_{g} \leq \int|h| \mathrm{e}^{-t H_{g}^{-w_{-}}} 1_{K_{n}} \mathrm{~d} \mu_{g} \leq\left\|\mathrm{e}^{-t H_{g}^{-w_{-}}}\right\|_{\mathrm{L}^{\infty}(M, g) \rightarrow \mathrm{L}^{\infty}(M, g)}\|h\|_{\mathrm{L}^{1}(M, g)}
$$

where we have used the self-adjointness of $\mathrm{e}^{-t H_{g}^{-w_{-}}}$for the first inequality, and the $q=\infty$ case for the inequality. Using monotone convergence this implies

$$
\left\|\mathrm{e}^{-t H_{g}^{-w_{-}}} h\right\|_{\mathrm{L}^{1}(M, g)} \leq C\left(w_{-}, t, g\right)\|h\|_{\mathrm{L}^{1}(M, g)} .
$$

We have shown so far that

$$
\left\|\mathrm{e}^{-t H_{g}^{-w_{-}}}\right\|_{\mathrm{L}^{1}(M, g) \rightarrow \mathrm{L}^{1}(M, g)},\left\|\mathrm{e}^{-t H_{g}^{-w_{-}}}\right\|_{\mathrm{L}^{\infty}(M, g) \rightarrow \mathrm{L}^{\infty}(M, g)} \leq C\left(w_{-}, t, g\right) .
$$

In case $1<q<\infty$, applying Riesz-Thorin's theorem with $T=\mathrm{e}^{-t H_{g}^{-w_{-}}}, a_{0}=b_{0}=1$, $a_{1}=b_{1}=\infty, C_{0}=C_{1}=C\left(w_{-}, t, g\right), r=1-1 / q$ we get

$$
\left\|\mathrm{e}^{-t H_{g}^{-w_{-}}}\right\|_{\mathrm{L}^{q}(M, g) \rightarrow \mathrm{L}^{q}(M, g)} \leq C\left(w_{-}, t, g\right),
$$

which completes the proof.

The essential point of Proposition A.1 is that the bound is of the form $\delta \mathrm{e}^{t C_{\delta}}$ and not simply $C_{1} \mathrm{e}^{t C_{2}}$ (which corresponds to a weaker "contractive Dynkin" assumption on $w_{-}$; cf. [19]), and precisely this stronger bound has been used with $q=\infty$ recently in the context of the Riemannian total variation in [8].

\section{REFERENCES}

[1] Aizenman, M. \& Simon, B.: Brownian motion and Harnack inequality for Schrödinger operators. Comm. Purre. Appl. Math. 35 (2) (1982), 209-273.

[2] Braverman, M. \& Milatovich, O. \& Shubin, M.: Essential self-adjointness of Schrödinger-type operators on manifolds. Russian Math. Surveys 57 (2002), no. 4, 641-692.

[3] Elworthy, K. D.: Stochastic differential equations on manifolds. London Mathematical Society Lecture Note Series, 70. Cambridge University Press, Cambridge-New York, 1982.

[4] Enciso, A.: Coulomb Systems on Riemannian Manifolds and Stability of Matter. Ann. Henri Poincare 12 (2011), 723-741.

[5] Grigor'yan, A: Estimates of heat kernels on Riemannian manifolds. Spectral theory and geometry (Edinburgh, 1998), 140-225, London Math. Soc. Lecture Note Ser., 273, Cambridge Univ. Press, Cambridge, 1999.

[6] Grigor'yan, A.: Heat kernel and analysis on manifolds. AMS/IP Studies in Advanced Mathematics, 47. American Mathematical Society, Providence, RI; International Press, Boston, MA, 2009.

[7] Grummt, R. \& Kolb, M.: Essential selfadjointness of singular magnetic Schrödinger operators on Riemannian manifolds. J. Math. Anal. Appl. 388 (2012), no. 1, 480-489. 
[8] Güneysu, B. \& Pallara, D.: Functions with bounded variation on a class of Riemannian manifolds with Ricci curvature unbounded from below. Math. Ann. 363 (2015), no. 3-4, 1307-1331.

[9] Güneysu, B.: Nonrelativistic Hydrogen type stability problems on nonparabolic 3-manifolds. Annales Henri Poincaré 13 (2012), 1557-1573.

[10] Güneysu, B.: Kato's inequality and form boundedness of Kato potentials on arbitrary Riemannian manifolds. Proc. Amer. Math. Soc. 142 (2014), no. 4, 1289-1300.

[11] Güneysu, B. \& Post, O.: Path integrals and the essential self-adjointness of differential operators on noncompact manifolds. Math. Z. 275 (2013), no. 1-2, 331-348.

[12] Güneysu, B.: On generalized Schrödinger semigroups. J. Funct. Anal. 262 (2012), 4639-4674.

[13] Kato, T.: Schrödinger operators with singular potentials. Israel J. Math. 13 (1972).

[14] Kuwae, K. \& Takahashi, M.: Kato class measures of symmetric Markov processes under heat kernel estimates. J. Funct. Anal. 250 (2007), no. 1, 86-113.

[15] Lieb, E.H. \& Seiringer, R.: The stability of matter in quantum mechanics. Cambridge University Press, Cambridge, 2010.

[16] Li, P. \& Wang, J.: Mean value inequalities. Indiana Univ. Math. J. 48 (1999), no. 4, $1257-1283$.

[17] Liao, M.: Factorization of diffusions on fibre bundles. Trans. Amer. Math. Soc. 311 (1989), no. 2, 813-827.

[18] Ouhabaz, E.-M. \& Stollmann, P. \& Sturm, K.-T. \& Voigt, J.: The Feller property for absorption semigroups. J. Funct. Anal. 138 (1996), no. 2, 351-378.

[19] Stollmann, P. \& Voigt, J.: Perturbation of Dirichlet forms by measures. Potential Anal. 5 (1996), no. $2,109-138$.

[20] Sturm, K.-T.: Schrödinger semigroups on manifolds. J. Funct. Anal. 118 (1993), no. 2, 309-350. 